\title{
The Effect of Using Nutritional and Herbal Supplements on Uterine Fibroids as an Alternative Community Based Management in Kisumu
}

\author{
Susan Aruwa ${ }^{1, *}$, Andrew Opondo Otieno ${ }^{2}$, Francis Owino Rew ${ }^{3}$ \\ ${ }^{1}$ Department of Community Health and Development, Great Lakes University of Kisumu, Kenya \\ ${ }^{2}$ Uzima University College, Kenya \\ ${ }^{3}$ Jaramogi Oginga Odinga University of Science and Technology, Kenya
}

Copyright $\mathrm{C} 2017$ by authors, all rights reserved. Authors agree that this article remains permanently open access under the terms of the Creative Commons Attribution License 4.0 International License

\begin{abstract}
For a long time, community based alternative methods of treating illnesses/conditions has not been given much weight that it deserves, as it is also contributing considerably towards health care and many people in the communities are using the alternative ways. In Kenya nearly $80 \%$ of the populations including the Maasai are still dependent on herbal medicine; it remains an important source of treatment, particularly for rural communities. The World Health Organization's global strategy includes popularizing and incorporating herbal medicine in the national health systems of member countries. However, knowledge on community based alternative management of fibroids is still not well understood by many in the population. The purpose of the study was to determine the various methods of alternative fibroid management using nutritional and herbal supplements at community level and their effectiveness at eliminating uterine fibroids. The main objective of the study was to find out the types of community based nutritional and herbal supplements that women use to manage fibroids. There was therefore the need to find out the activities of the alternative medicine practitioners with specific interest on nutritional and indigenous herbal supplements treatment and determine whether the supplements and herbal medicines being administered were effectively eliminating uterine fibroids. There was an existing gap in the naming of the local herbs being used, quantities of drug being administered, the ratios of different drugs mixed in a given concoction, effectiveness and documentation of the local herbs used for alternative management of fibroids in this country.
\end{abstract}

Keywords Nutritional and Herbal supplements, Alternative Management, Uterine Fibroids, Women

\section{Introduction}

In developing countries with poor infrastructure, traditional healers are located in nearly every rural village setting as well as in the busy urban areas. Therefore, they are a godsend to Africa's cash-strapped health ministries since they are already on the ground. However, despite their knowledge and popularity, traditional healers have been given a raw deal by Western medical experts. True collaboration, requires a measure of respect for indigenous medicine and African culture generally. It also requires the shedding of stereotypes of African traditional healers [18] most important of all, is the search for a common ground between western biomedicine and traditional healers and building upon that common ground.

\section{Epidemiology}

Fibroids are among the most common conditions affecting women of reproductive age and especially the older women. They are the most common tumours in women of reproductive age. Fibroids most commonly tend to affect middle aged women, 70 to 80 percent of women will have had one by the age of fifty, although most are too small to cause symptoms [9].

Problems associated with fibroids include heavy menstrual bleeding occurring throughout the cycle, pain in the pelvic area increase in uterine size, anemia, and pressure on the lower abdomen with increased urine frequency. A fibroid may become malignant. They also cause complicated pregnancies and mechanical distortion causing sub fertility or occlusion of the fallopian tubes. An abdominal cavity grossly distorted with fibroids usually prevents implantation of a fertilized ovum. While fibroids rarely progress to malignancy, in symptomatic cases, they can lead to multiple gynecological problems such as pelvic pain, infertility, menstrual aberrations and spontaneous abortions, significantly affecting the quality of life among women. Although these benign tumours represent a significant public health concern, though their epidemiology is poorly 
understood [13]

\section{Exposure and Diagnosis}

Estimating the overall prevalence of women with fibroids in the population is difficult, since estimates will vary depending on the population examined and the methods used to detect and diagnose fibroid prevalence in asymptomatic women. A total of 20 to $25 \%$ of reproductive age women have clinically symptomatic fibroids. The true population of fibroids is probably underestimated because of the unknown distribution of subclinical tumours. Studies screening randomly selected women using ultrasonography or pathologic examination of uteri have reported uterine Leiomyoma prevalence values ranging from 5.4 to $77 \%$ in pathological studies of hysterectomy specimens performed for all indications not limited to fibroids alone, fibroids were detected in 45 to $77 \%$ of the specimens. Fibroid symptoms are the most common indication for 30 to $40 \%$ of all hysterectomies in the U.S.A or 150 to 200,000 hysterectomies and at least 34,000 myomectomies annually. [4]

\section{Risk Factors Influencing the Development of Fibroids}

Although the exact cause of uterine fibroids is unknown, it's thought that certain genetic and hormonal factors may play a role in their development. For instance, the hormones estrogen and progesterone may stimulate the growth of uterine fibroids. The following may increase the risk for uterine fibroids: obesity, family history of uterine fibroids and not having given birth [5].

\section{Pathogenesis}

It is not clear whether diet habits, such as consuming red meat, ham, green vegetables, or fiber, could influence the growth of myomas. It is also difficult to analyze the specific effects of physical exercise on the development of uterine myomas, as only a few observational studies have addressed this aspect so far [4]. Although the exact cause of uterine fibroids is unknown, it is thought that certain genetic and hormonal factors may play a role in their development. For instance, the hormones estrogen and progesterone may stimulate the growth of uterine fibroids. The following may increase one's risk for uterine fibroids: obesity, family history of uterine fibroids, not having given birth [20]

\section{Therapy}

With alternative therapies, the herbal remedies are reported to slowly reduce the size of fibroids and control the further growth of new ones [20]. Natural herbal remedies can be a better alternative choice. Since hormonal imbalance is a likely cause of fibroid growth, methods to restore hormonal balance can be helpful in reducing the symptoms of fibroids and their size. There are many techniques in alternative medicine that may be helpful. Herbal teas are one of the best methods that can be used for the treatment of uterine fibroids [10]. The main components of natural treatment for fibroids were: Detoxification, Internal body cleansing, extensive dietary modification, an exercise regime, alternative therapies, weight management, and dietary supplementation. To be successful, it was important to attempt to eliminate the root causes of fibroids. The difficulty was that one could not know (with the exception of an obvious one such as bodyweight and, possibly the diet) which were responsible and to what extent they were influencing one's condition. This is why a robust "no stone unturned" approach was necessary to remove all the potential causes, thus allowing for natural shrinkage [19] Hormone Support a natural fibroids treatment compared to the usual medical techniques of treating this condition has no negative side effects. Previalin-HS is the second product in the Uterine Fibroid package. Previalin-HS was designed for women who are looking to take control of the harmful effects of estrogen dominance. Women that are suffering from Uterine Fibroids are unaware that unbalanced hormone levels can cause the growth of fibroids. Previalin-HS also address's many symptoms that are also caused from unbalanced hormones levels, if they are having trouble with pelvic pain, mood swings, bloating, depression and heavy periods Previalin-HS is a product that can help [1]

Green tea and uterine fibroids: In an animal study published in 2010, scientists discovered that 8 weeks of treatment with green tea extract led to a significant decrease in the volume and weight of uterine fibroids among a group of mice. In traditional Chinese medicine and uterine fibroids in a pilot study published in 2002, researchers assigned a group of women with uterine fibroids to 6 months of treatment with traditional Chinese medicine, body therapy (including bodywork), and guided imagery. Study results showed that fibroids shrank or stopped growing in twenty-two patients in the treatment group, compared with three patients in the control group [22].

It was very important to restore a woman's hormonal balance in order to reduce the symptoms and further growth of fibroids. This approach was combined with appropriate changes in diet and lifestyle in order to get its full benefits. It was highly advisable that women who had fibroids refrain from including foods that were rich in saturated fats alcohol and sugar in their everyday meals. Eating these types of foods had the tendency to overwork the liver and hindered the effective breaking down of hormones. Following a healing diet that the primary objective was to lessen the intake of estrogen heavy foods was very important [12].

The study received ethical review and was granted the permission to carry out the research in the indicated area by 
the following university board, GLUK Research Ethics Committee (GREC)

Ref: No. GREC 050/2011, Certificate of Approval of Research Protocol

To: SUSAN AKUMU ARUWA (Principal Investigator)

Date: Wednesday, July 25, 2012

Boniface Obondi, SECRETARY, GREC

\section{Study Objectives}

The study sought out

1. To determine the different community based alternative ways of nutritional and herbal supplements that women use to manage fibroids

2. To determine the measure of success the alternative ways have made in clearing the fibroids after having used the nutritional and herbal supplements to manage the fibroids?

3. To find out reasons why women turn to community based alternative management of fibroids.

\section{Specific Research Questions}

1. What are the various types of community based nutritional and herbal supplements that women use to manage fibroids?

2. What measure of success has the nutritional and herbal supplements made in clearing the fibroids?

3. What are the reasons making women turn to alternative ways of managing fibroids?

\section{Methodology}

This study used descriptive cross-sectional, design founded on quantitative approaches of data collection; it examined those affected by the disease in a defined population at a particular time. The data source was both primary and secondary, getting it from both the women who had been diagnosed with fibroids and the practitioners from Herbal medicine and Nutritional supplements clinics. Structural interview method was used on quantitative data. All the women clients attending the organized herbal medicine and nutritional supplements clinics health days were targeted for the interviews. It was estimated that the total number of women who attended the clinics would be 400. The exclusion criteria were those women with fibroid like symptoms in the communities but had not undertaken a confirmatory diagnosis at the health facilities for fibroids, while the inclusion criterion were women diagnosed with uterine fibroids at the health facilities or had a history of fibroids and had been diagnosed at the health facilities. Those who consented to participate were included and those who did not provide consent were not included.

\section{Study Area}

This study was carried out in the communities of Kisumu municipality; collecting data from the different categories of the social classes of women living within the municipality, the women were registered at the herbal and nutritional health clinics.

\section{Study Population}

The study population were women of reproductive age (15-49 years of age) who had a history of fibroids, whether recovered or not and resided within the urban population of Kisumu municipality and were registered at the herbal and nutritional health "clinics". The urban population of women of reproductive age in Kisumu Town East was 105,024 females at the time of the study. Literature Review states that $25 \%$ of women have uterine fibroids [3]; The women were accessed from a sampling frame obtained from Tianshi company, Tasly Company, Dynapharm Company, Forever Living, Anysee, Edmark, Indian herbs, Pellamic Herbal, Makini herbal, Foratram International and the local herbalists from the Jubilee and Kibuye Municipal markets.

\section{Sample Size Determination}

The sample size was calculated to be 347 women. The respondents were selected from all the registered nutritional supplement health facilities and herbal "clinics", they were then interviewed. All cases from the nutritional supplement health facilities and herbal "clinics" were interviewed until the required number of 350 was attained. The sample size was worked out from the basic formula of a model that had been used by Fishers.

\section{Data Collection Methods and Data Collection Tools}

Quantitative method of data collection was applied in this study. The questionnaire was divided into six sections. The first section was the identification page, followed by the second section which were closed ended questions seeking information on Socio-demographic factors, the third section sought information on methods of fibroid management of the study population these were both close and open ended questions, and Section four sought to find out the health facilities where clients went for fibroids diagnosis these were close ended questions. The fifth section sought information on fibroid clearance among patients who completed alternative management of fibroids these were both close and open ended questions. Section six sought to find out the reasons for preference of alternative fibroid management these were open ended questions.

The structured questionnaire was administered in all the 
registered nutritional supplement health facilities and herbal clinics on designated health days, for health talks organized by the facilities. Before the interviews were conducted, the health facilities managements were informed and they further informed their clients on the study to be conducted. The researcher briefed the clients on the purpose of the study during the meetings and explained that each client would be interviewed individually. After informed consent was obtained, the respondents were each interviewed. Interviews were done at all the herbal and nutritional supplements clinics.

The community health workers helped in mobilizing the women to attend the health days for the health talks conducted within the facilities on designated health days. The facilities normally held health talks on a regular basis with their clients. Multiple meetings were held and interviews were done on those days until a majority of the clients were interviewed. The community health workers mobilized the women who had recovered and those who were still on treatment and requested them to carry along their treatment cards (the treatment cards was a confirmatory measure that the women being interviewed actually undertook the treatment and the fibroids they had were cleared on completion of their treatment as recorded on their cards) while attending the organized health day talks.

\section{Data Processing, Analysis and Presentation}

The quantitative data was entered and processed both manually and electronically using the Statistical Package for Social Sciences (SPSS version 18). Data was analyzed using both the basic frequencies and proportion. Exploratory data analysis was done employing both descriptive and inferential statistics. Associations between variables were done through cross tabulations, and measures of associations using chi-square test which was used to determine the strength of the associations. Statistically significant results was measured at $\mathrm{p}$ value $<0.05$. Qualitative data was recorded on note books and contents analysis was adopted where the data was organized into themes associated with specific objectives. Sample of opinions of the key informants were reported verbatim to emphasize its importance. Quantitative data are presented in tables and charts and are interpreted as per the findings of the study.

\section{Findings}

The results were coded and entered into a window created in SPSS version 18. The data are presented using frequency tables and charts. Chi square tests were used to assess association between variables with odds ratio and $p$ values being reported. A p-value $<0.05$ was considered statistically significant result.

A total of $35.4 \%$ of women used unprocessed indigenous herbs, $33.1 \%$ used supplements, and $25.7 \%$ used locally processed indigenous herbs. Of the 350 women, $9.4 \%$ had fibroids completely removed from their bodies. Those who used food supplements had significantly higher fibroid clearance rates compared to those who used other herbal alternatives $(\mathrm{p}<0.0001)$.

The result indicated that of the 350 women interviewed; the number of women who mentioned that fibroids were not completely cleared from their bodies was $90.6 \%$. However, the distribution in proportion of women whose fibroids were completed cleared tended to gradually increase by increasing age, between 15-34 years and again from 40 years and above. The results indicate that out the women who were aged 15-19 years, $0.3 \%$ of them had fibroids completely cleared from their bodies compared to $2.0 \%$ of those in the $40-44$ and 45-49 years age groups whose fibroids also got completely cleared.

The indigenous herbs that were mostly used by the women in Kisumu municipality had not been given particular attention, research had not been done on the locally unprocessed indigenous herbs; neither systematic reviews nor clinically tested trials had been done on the indigenous and available nutritional and herbal supplements used in the communities in Kisumu, Kenya.

Table 1. Demographic Characteristics of the Study Population

\begin{tabular}{|c|c|c|c|c|c|c|c|c|c|c|c|}
\hline \multicolumn{2}{|c|}{ AGE in $\%$} & \multicolumn{2}{|c|}{ Marital Status in \% } & \multicolumn{2}{|c|}{ Education in $\%$} & \multicolumn{2}{|c|}{ Income in $\%$} & \multicolumn{2}{|c|}{ Ethnicity in $\%$} & \multicolumn{2}{|c|}{ Religion in \% } \\
\hline $15-19$ & 4.9 & Married & 29.4 & Secondary & 20 & Self Employed & 28.9 & Luo & 19.2 & Christianity & 66 \\
\hline $20-24$ & 8.0 & Single & 28.6 & High School & 21.4 & Farming & 20.0 & Luhya & 18.0 & Muslim & 30.9 \\
\hline $25-29$ & 14.3 & Widows & 29.7 & Diploma & 16.9 & Salaried & 23.4 & Kisii & 15.1 & Others & 3.1 \\
\hline $30-34$ & 20.0 & Divorced & 12.3 & Graduate & 10.3 & Remittances & 14.9 & Kuria & 15.4 & - & - \\
\hline $35-39$ & 20.3 & - & - & Primary & 16.6 & No income & 8.6 & Kalenjin & 13.7 & - & - \\
\hline $40-44$ & 19.1 & - & - & $\begin{array}{l}\text { No formal } \\
\text { Education }\end{array}$ & 9.7 & Others & 4.2 & Kikuyu & 12.9 & - & - \\
\hline $45-49$ & 13.4 & - & - & Post Grad & 5.1 & - & - & Others & 5.7 & - & - \\
\hline \multicolumn{2}{|c|}{ Total } & \multicolumn{2}{|c|}{100} & \multicolumn{2}{|l|}{100} & \multicolumn{2}{|l|}{100} & \multicolumn{2}{|c|}{100} & \multicolumn{2}{|c|}{100} \\
\hline
\end{tabular}

Table 1 above gives a summary of the demographic characteristics of the study population. 


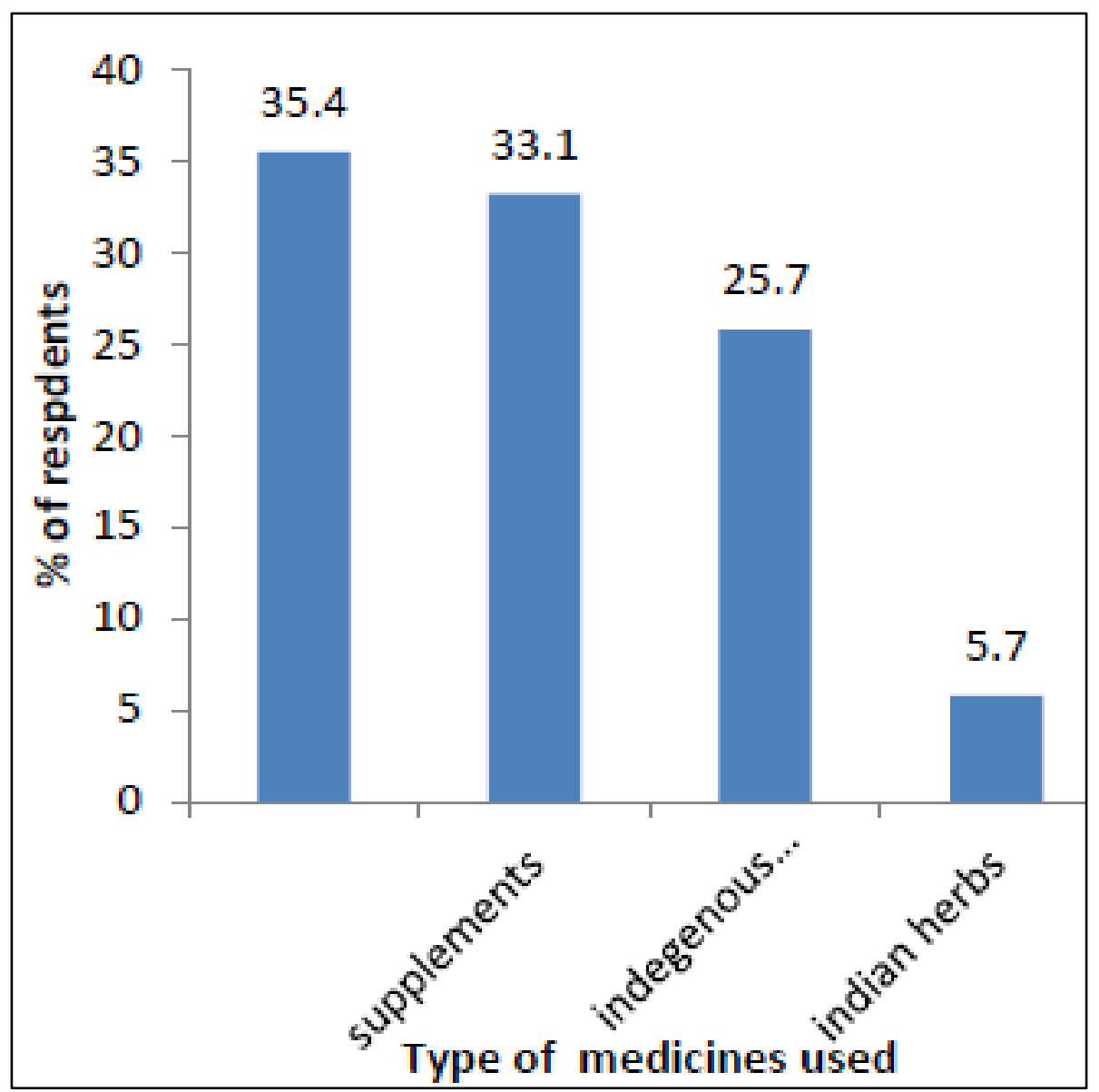

Figure 1. Summaries of types of herbal medicines used in management of fibroids

Figure 1 above shows the summary of types of herbal supplements used in alternative management of fibroids at community level and the proportion of the respondents interviewed. The results indicate that $35.4 \%$ utilized unprocessed indigenous herbs, $33.1 \%$ used supplements, $25.7 \%$ used indigenous processed herbs and $5.7 \%$ consumed Indian herbs. The unprocessed indigenous herbs had the highest percentage of consumption followed by nutritional supplements.

Table 2. Distribution of women by age group whose fibroids were completely cleared

\begin{tabular}{|c|c|c|c|c|c|c|c|c|}
\hline Age group of women & $15-19$ & $20-24$ & $25-29$ & $30-34$ & $35-39$ & $40-44$ & $45-49$ & Total \\
\hline Percentage of fibroid clearance & 0.3 & 0.6 & 1.4 & 1.7 & 1.4 & 2.0 & 2.0 & 9.4 \\
\hline
\end{tabular}

Table 2 indicates the percentage distribution of those whose fibroids had been cleared by their different age groups. The study sought to determine degree of achievement of fibroid clearance by the alternative management using nutritional and herbal supplements.

\section{Discussion on Controversies}

Controversies that face the successes that nutritional and herbal supplements have made at clearing fibroids include claims such as these; in the medical world, the only successful management for fibroids is surgery. The two types of surgery are myomectomy, which only removes the existing fibroids, and hysterectomy, which removes the entire uterus [13].
Other statements are like this one; "Many alternative practitioners and women who use alternative medicine have reported individual case histories that create some hope for reducing the size of fibroids'. The reporter sites cases where the fibroid growths and the size of the uterus have been significantly reduced. The problem is that the results are very inconsistent and random. Often the cases that have shown the most dramatic improvements are the women who are in their late forties and early fifties and are in fact almost menopausal or in fact menopausal[20].

Some remarks discouraging women from taking herbs are; "Herbal remedies are potent medicines and can interact with other medications and cause side effects; inform your doctor of all herbal remedies you are using, women who take synthetic hormones have an increased risk, as hormones may promote fibroid growth. This occurs because fibroids contain 
high concentrations of estrogen receptors than normal uterine tissue. Lack of documented use of herbal medicine for fibroid management and unregulated use of the alternative therapy, inhibits planning, managing, and regulating its practitioners.

Despite all these controversies, from studies of other authors as given in literature review we find that in China, for instance, herbal treatment has attained a high level of scientific and technological formulation, for instance In traditional Chinese medicine and uterine fibroids in a pilot study published in 2002, researchers assigned a group of women with uterine fibroids to six months of treatment with traditional Chinese medicine, body therapy (including bodywork), and guided imagery. Study results showed that fibroids shrank or stopped growing in twenty-two patients in the treatment group, compared with three patients in the control group [21].

\section{Recommendations}

1. A government board that deals with registration, regulation and control of the practices and activities of herbal practitioners should be put in place in Kenya to check on the safety of the supplements being administered and help practitioners establish standard herbal supplement utilization.

2. The indigenous herbs that were widely used by the women should be given special attention and more research done on them by using systematic reviews and clinically tested analysis so that there can be records of success stories on them as there were for nutritional supplements from both the western and eastern nations.

3. Advocacy for behavior change in use of supplements and processed local herbs that are successful in clearing fibroids should be carried out at higher levels of Health Service provision.

\section{Conclusion}

The study established that fibroids can be completely eliminated using nutritional and herbal supplements. Of the 350 women interviewed $9.4 \%$ had fibroids completely cleared from their uterus. Complete clearance of fibroids for those who used nutritional supplements was with $6.2 \%$, while those who used indigenous processed herbs were by $3.1 \%$.

\section{Dedication}

This research is dedicated to all the women who have suffered from fibroids and those who are still having fibroids and do not want surgery and other invasive methods of management, but are looking for alternative less invasive ways to remove the fibroids completely, without the complications that surgery presents to those who have undertaken it.

\section{REFERENCES}

[1] Amanda Leto (Aug 05, 2010). Fibroids Miracle, the only clinically proven 3 step holistic system for eliminating uterine fibroids naturally, published by Gail Atkinson, SAN Diego, California, USA

[2] Amanda Leto (Aug 05, 2010). Uterine Fibroids 14-Day Meal Plan \& Recipes, Real estate Religion Reports R

[3] Baird Day D, Dunson DB, Hill MC, Cousins D and Schectman JM (2003). High Cumulative incidence of uterine leiomyoma in black and white Women: Ultrasound evidence. American Journal of Obstetrics and Gynecology. 188:100. Elsevier B. V. Publication, Oxford

[4] Evans P, Brunsell S (2007). Uterine fibroid tumors: Diagnosis and treatment. American Family Physician 75 10: U.S.A

[5] Faerstein E, Szklo M., Rosenshein, N., (2001). Risk factors for uterine leiomyoma: A practice-based case control study. I African-American heritage, reproductive history, body size, and smoking; American Journal of Epidemiology; 153:1

[6] Holmes M (2010). Weight Loss, Phytotherapy like our Herbal Equilibrium, D\&C, hysteroscopy, restore hormonal balance nutritional guidelines, Women's Health NP Philadelphia, PA: Harcourt Publishers Ltd;

[7] Hopkins (2010), “The John Hopkins Family Health Book. The Essential Home Medical Reference the help you and your family" Collins Living Publishers

[8] Kokwaro J, Johns T, (1998), Luo biological dictionary, East African Educational Publishers Limited. ISBN9966 468412

[9] Lee T K, Lee DK, Kim DI, Lee YC, Chang YC, Kim CH (2004). "Differential inhibition of Scutellaria barbata D. Don (Lamiaceae) on HCG-promoted proliferation of cultured uterine leiomyomal and myometrial smooth muscle cells "Journal of Immunopharmacol Immunotoxicol 26(3):329-42. Keats Publishing Inc., USA.

[10] Mehl-Madrona L (2002). "Complementary medicine treatment of uterine fibroids: a pilot study. "Alternative, the Health Medical Journal 8(2):34-6, 38-40, 42, 44-6. Tucson AZ 85715

[11] Noad T, Birnie A, (1989) Trees of Kenya, Publisher, T. C Noad and A. Birnie, Nairobi, Kenya, General Printers Limited

[12] Rockville (April 29, 2009.)Management of uterine fibroids: An update of the evidence. Publisher, Agency for Healthcare Research and Quality, London, UK: BMJ

[13] Rodriguez M I, Warden M, Darney P D, (2012): Intrauterine Artery Embolization with Surgical Treatment, Hindawi Publishing cooperation

[14] Stewart EA. (April, 2009). Epidemiology, clinical manifestations, diagnosis, and natural history of uterine leiomyomas Publisher, Institute of Psychiatry, King's College 
London, UK, SE5 8AF

[15] Stewart E, A. (April, 2009) Pathogenesis of uterine leiomyomas Publisher, Institute of Psychiatry, King's College London, UK, SE5 8AF

[16] Teel W, Hirst T. (1990) A Pocket Directory of Trees and Seeds in Kenya, publisher Kenya Energy Non-Governmental Organisation Nairobi, Kenya. , General Printers Limited

[17] University of Maryland Medical Centre publication (2011)

[18] UNEP (2009), Kenya Forestry Research Institute survey on Environmental Health, publisher Government of Kenya
[19] Warshowsky A, Oumano E (2009).Healing Fibroids: A Doctor's Guide to a Natural Cure, Publisher: Fireside Books, Pocket. ISBN 9780743418256

[20] Wong C (2010), Journal of Alternative and Complimentary Medicine, Fairwinds Press

[21] Zhang Y, Peng W, Clarke J, Liu Z. (2010) "Acupuncture for uterine fibroids" Cochrane Database Systematic Review $20 ;(1): C D 007221$.

[22] Zhang D, (2010). "Green tea extract inhibits proliferation of uterine leiomyoma cells in vitro and in nude mice." American Journal Obstet Gynecology 12. 